\title{
Análise dos indicadores da COVID-19 no Nordeste brasileiro em quatro meses de pandemia
}

\section{Analysis of COVID-19 indicators in the Northeast region of Brazil in the four first months of the pandemic}

\author{
Bruno Quintela Souza \\ de Moraes',* iD \\ Isa Carolina Gomes Félix'
}

Túlio Romério Lopes Quirino" (iD

Michael Ferreira Machado' iD
Universidade Federal de Alagoas (UFAL), Maceió, AL, Brasil

" Universidade Federal de Pernambuco (UFPE), Recife, PE, Brasil

\footnotetext{
* E-mail: bruno.moraes@arapiraca.ufal.br
}

\section{RESUMO}

Introdução: O novo coronavírus, ou SARS-CoV-2, surgiu no fim de 2019 na China e se disseminou rapidamente, resultando num surto mundial. A pandemia da COVID-19, como foi chamada a doença causada pelo vírus, foi declarada pela Organização Mundial da Saúde em março e ultrapassou a marca de um milhão de infectados e de 50 mil mortos no Brasil após quatro meses do primeiro caso. Objetivo: Analisar a evolução dos indicadores sobre a COVID-19 no Nordeste do Brasil e correlacioná-la ao nível de isolamento social registrado em cada estado nordestino. Método: Trata-se de um estudo transversal com dados coletados a partir de painéis epidemiológicos do Ministério da Saúde e da Universidade de São Paulo acerca do número de casos e óbitos e, também, da empresa de geolocalização Inloco que calculou os níveis de isolamento social no Brasil. As variáveis analisadas foram: taxa de incidência, taxa de mortalidade, coeficiente de letalidade e índice de isolamento social. Resultados: Os indicadores são interdependentes e há certa correlação entre os níveis de isolamento social e as taxas de incidência e mortalidade, sendo correlações fracas ou moderadas. Conclusões: As diferenças entre os estados nordestinos, principalmente as sociais, refletem em seus indicadores e no impacto da pandemia em cada um deles, sendo um desafio posto aos gestores de saúde.

PALAVRAS-CHAVE: Pandemia; Coronavírus; Infecções por Coronavírus; Indicadores de Saúde

\begin{abstract}
Introduction: The novel coronavirus or SARS-CoV-2 came up in the end of 2019 in China and quickly spread, resulting in a global outbreak. The COVID-19 (name given to the disease caused by the virus) pandemic was declared by WHO in March and surpassed the mark of one million cases and fifty thousand deaths in Brazil after four months since the first case was confirmed. Objective: The paper aimed to analyze the evolution of COVID-19 indicators in the Brazilian Northeast and correlate it to the social distancing levels in each northeastern state. Method: It is a traversal study with data collected from epidemiological panels of the Health Ministry of Brazil and the University of São Paulo about cases and deaths numbers and from the geolocation's company Inloco that calculated the social distancing levels. The analyzed variables were incidence rate, mortality rate, lethality coefficient and social distancing index. Results: The indicators are interdependent and there is certain correlation between the social distancing levels and incidence and mortality rates - they were weak or moderate. Conclusions: Differences between northeastern states, mainly social ones, reflect in their indicators and in the pandemic impact in each of them. It is a challenge proposed for health gestors.
\end{abstract}

KEYWORDS: Pandemics; Coronavirus; Coronavirus Infections; Health Status Indicators 


\section{INTRODUÇÃO}

O fenômeno da globalização acompanha o desenvolvimento tecnológico das últimas décadas, derrubando, assim, barreiras físicas existentes entre os continentes ${ }^{1}$. É nesse contexto que emerge, pela quinta vez, um vírus da família Coronaviridae, causando surto entre os seres humanos ${ }^{2}$.

O novo coronavírus, denominado SARS-CoV-2, surgiu na cidade chinesa de Wuhan, no final de 2019, e logo se disseminou por vários países. A COVID-19, doença causada por este novo vírus, foi declarada como pandemia pela Organização Mundial da Saúde (OMS) no dia 11 de março de 20203. Contudo, antes mesmo desta declaração, o Brasil já havia confirmado seu primeiro caso, no dia 25 de fevereiro de 2020 e, desde então, o número de casos demonstrou crescimento exponencial com o registro de 1.313 .667 casos confirmados e de 57.070 óbitos até 27 de junho de $2020^{4}$.

Do ponto de vista clínico, a evolução da COVID-19 varia muito entre os indivíduos infectados; desde pessoas assintomáticas a quadros de pneumonia grave e morte. Cerca de $80 \%$ dos infectados apresentam um quadro leve da doença e os desfechos mais críticos se dão em pequena parcela das pessoas infectadas, aproximadamente $2 \% 5,6$. A transmissão do SARS-CoV-2 acontece pessoa a pessoa, através do contato com gotículas respiratórias ou por meio de objetos contaminados ${ }^{6,7}$.

Como resultado da alta transmissibilidade, aliada à falta de medicamentos e vacinas específicos, mesmo com a baixa letalidade da COVID-19, tem-se o agravamento da crise sanitária global com maior número absoluto de óbitos ${ }^{1,7}$. Buscando atenuar a situação, que varia entre as diferentes localidades, autoridades sanitárias e estruturas locais de governo propõem intervenções não farmacológicas como ações de enfrentamento, o que envolve a adoção de medidas (1) individuais: uso de máscaras de proteção facial, higienização constante das mãos e adoção da etiqueta respiratória; (2) ambientais: higienização de locais públicos como elevadores e halls de edifícios; e (3) comunitárias: proibição de aglomerações, fechamento de locais com grande fluxo de pessoas - parques e shoppings, por exemplo - e isolamento social ${ }^{7,8,9}$.

O isolamento social tem como principal objetivo reduzir as interações em uma comunidade - na qual estão inclusos infectados confirmados e aqueles não identificados que não se encontravam isolados. Esse tipo de intervenção é necessário para o achatamento da curva epidemiológica, evitando a transmissão desenfreada e a superlotação do sistema de saúde, que ganha tempo para se estruturar ${ }^{6}$. Mas, para ser efetiva, essa medida depende de vários fatores, principalmente, sociais, culturais e econômicos ${ }^{6,8}$.

Em face da emergência da pandemia ocasionada pelo vírus SARS-CoV-2, este estudo se faz necessário por possibilitar informações que auxiliam no planejamento de estratégias para se enfrentar o atual cenário ${ }^{10}$. Isto posto, o artigo objetivou analisar a evolução dos indicadores da pandemia da COVID-19 nos estados da região Nordeste do Brasil e correlacioná-la aos níveis de isolamento social nessas localidades, considerando as 13 primeiras semanas da epidemia no país.

\section{MÉTODO}

Trata-se de um estudo transversal que analisa a evolução dos indicadores, semana a semana, sobre a COVID-19 no Nordeste do Brasil. Os dados foram coletados a partir dos painéis epidemiológicos do Ministério da Saúde (MS) ${ }^{4}$ e da Universidade de São Paulo (USP) ${ }^{11}$, compreendendo o período de 26 de fevereiro a 24 de junho de 2020.

O Nordeste, região mais afetada pela epidemia do novo coronavírus, é composto por nove estados: Alagoas, Bahia, Ceará, Maranhão, Paraíba, Pernambuco, Piauí, Rio Grande do Norte e Sergipe. A estimativa da população residente no país, no Nordeste e em cada estado, bem como em suas respectivas capitais foi coletada do Instituto Brasileiro de Geografia e Estatística (IBGE) ${ }^{12}$.

Além disso, os índices de isolamento social dos estados nordestinos foram catalogados a partir do Mapa Brasileiro da COVID-19, desenvolvido pela empresa brasileira de tecnologia e geolocalização Inloco (https://inloco.com.br/) ${ }^{13}$. Esta garante o anonimato dos usuários durante a captura dos dados.

A partir da coleta, os dados - número de casos confirmados, óbitos confirmados, índice de isolamento social e população residente - passaram a ser tabulados, por semana, por meio do Microsoft Office Excel 2013. Foram calculados os seguintes indicadores: a) taxa de incidência por 100 mil habitantes (casos confirmados $\div$ população residente $\times 100.000$ ); b) taxa de mortalidade por 100 mil habitantes (óbitos confirmados $\div$ população residente $\times 100.000$ ); e c) coeficiente de letalidade (óbitos confirmados $\div$ casos confirmados $\times 100$ ).

Além disso, foi utilizado o software Statistical Package for the Social Sciences (SPSS), versão 25, para verificar a existência de correlação entre as taxas de incidência e de mortalidade com os índices de isolamento social registrados durante o período. Para tanto, foram realizados o teste exato de Fisher, o teste do qui-quadrado e a correlação de Spearman e, para ser significante, o p-valor deve ser menor que 0,05. As correlações foram interpretadas através do seu coeficiente de correlação $(\rho)^{14}$.

O presente estudo se utiliza de dados de domínio público e livre acesso, sem a identificação dos participantes. Não sendo necessária, portanto, a apreciação no Sistema Comitê de Ética em Pesquisa/Comissão Nacional de Ética em Pesquisa (CEP/Conep), em conformidade com as Resoluções $n^{\circ} 466$, de 12 de dezembro de 2012; n 510, de 7 de abril de 2016 e n ${ }^{\circ} 580$, de 22 de março de 2018, do Conselho Nacional de Saúde, que regulamentam as pesquisas com seres humanos e no âmbito do Sistema Único de Saúde, no Brasil. 


\section{RESULTADOS}

Entre 26 de fevereiro, dia em que ocorreu a notificação do primeiro caso confirmado de COVID-19 no Brasil, e 24 de junho de 2020 , foram registrados 1.188 .631 casos e 53.830 óbitos em decorrência da infecção pelo novo coronavírus, com base nos dados do MS e do Painel Epidemiológico da USP. Nesse mesmo recorte temporal, o Nordeste contabilizou 408.341 casos (34,4\%) e 17.248 óbitos (32,0\%), sendo, até então, a região do país mais acometida pela pandemia.

Primeiramente, ao se analisar os dados brutos e traçar as demais variáveis, foi possível observar que a região Nordeste possui taxas de incidência e mortalidade por 100 mil habitantes $(718,5$ e 30,3 , respectivamente) maiores que as do Brasil (565,6 e 25,6, respectivamente). Ainda, destaca-se que nos estados com maior número de casos - Ceará e Maranhão - a taxa de incidência é muito maior do que a média da região e do país (Tabela 1 ).

Em se tratando da letalidade da COVID-19, no período estudado, a taxa brasileira foi de $4,5 \%$ e a da região Nordeste foi um pouco menor, $4,2 \%$. Vale salientar que quase todos os estados possuem coeficientes de letalidade menores do que os encontrados nos níveis nacional e regional; as exceções são os estados do Ceará e de Pernambuco, que apresentaram os maiores indicadores. Paraíba é a unidade federativa do Nordeste com a menor letalidade (Figura).

Os índices de isolamento social registrados pela Inloco se mostram semelhantes tanto ao nível nacional, quanto na região Nordeste, $39,0 \%$ e $41,5 \%$, respectivamente. Somente Alagoas, Paraíba, Pernambuco e Sergipe apresentaram isolamento social maior do que as médias regional e nacional. 0 estado do Maranhão apresentou o menor índice no período.

Dentre os indicadores acima, a Bahia dispõe de um bom panorama. Mesmo sendo o estado mais populoso da região, com mais de 15 mil casos confirmados e uma das menores médias do índice de isolamento social, registrou as menores taxas de incidência e mortalidade do Nordeste e seu coeficiente de letalidade encontra-se abaixo dos coeficientes regional e nacional.

Já nas análises realizadas a partir do SPSS, que levaram em conta o índice de isolamento social de cada localidade, no teste qui-quadrado de Pearson, as variáveis não são dependentes dentro do período, pois apresentam o $\mathrm{p}$-valor maior que 0,05 .

$\mathrm{Na}$ correlação de Spearman, considerando o p-valor das análises, nenhum estado mostrou significância nas correlações entre incidência/mortalidade e nível de isolamento social. Contudo, a interpretação do coeficiente de correlação ${ }^{14}(\rho)$ pode nos ajudar a compreender a relação entre as variáveis, já que, na função traçada pelo software, os valores variam em uma faixa estreita, entre -1 e 1 (Tabela 2). Quanto mais próximo dos extremos, mais fortes são as correlações; e quanto mais próximo de zero, mais fracas elas são.

Sendo assim, existe fraca correlação entre a incidência e os níveis de isolamento social na maioria dos estados nordestinos, com exceção de Bahia, Maranhão e Piauí - correlações muito fracas - e da Paraíba, que apresentou correlação moderada. Já no tocante aos testes entre as variáveis taxa de mortalidade e nível de isolamento social, as correlações são fracas em Alagoas, Paraíba, Pernambuco e Sergipe. Nos demais estados as correlações são muito fracas.

\section{DISCUSSÃO}

A nova dinâmica socioeconômica, globalizada, com grande fluxo de pessoas e objetos, facilitou a disseminação da COVID-19, causando impacto global de alcance e velocidade nunca vistos $^{15}$. Nessa perspectiva pandêmica, o Brasil passa por uma crise sanitária sem precedentes, agravada por um panorama político e econômico também em estado crítico ${ }^{1,16}$. Isso porque há um

Tabela 1. Taxas de incidência e mortalidade por 100 mil habitantes e coeficiente de letalidade (\%) no Brasil, na região Nordeste e seus estados até 24 de junho de 2020.

\begin{tabular}{|c|c|c|c|}
\hline Recorte geográfico & $\begin{array}{c}\text { Taxa de incidência } \\
\text { (casos/100 mil habitantes) }\end{array}$ & $\begin{array}{c}\text { Taxa de mortalidade } \\
\text { (óbitos/100 mil habitantes) }\end{array}$ & $\begin{array}{c}\text { Coeficiente de letalidade } \\
\text { (\%) }\end{array}$ \\
\hline Brasil & 565,6 & 25,6 & 4,5 \\
\hline Nordeste & 718,5 & 30,3 & 4,2 \\
\hline Alagoas & 935,8 & 28,1 & 3,0 \\
\hline Bahia & 349,2 & 10,4 & 3,0 \\
\hline Ceará & $1.090,4$ & 63,7 & 5,8 \\
\hline Maranhão & $1.036,2$ & 25,9 & 2,5 \\
\hline Paraíba & 993,3 & 20,6 & 2,1 \\
\hline Pernambuco & 565,3 & 46,3 & 8,2 \\
\hline Piauí & 521,8 & 16,7 & 3,2 \\
\hline Rio Grande do Norte & 646,3 & 24,5 & 3,8 \\
\hline Sergipe & 885,4 & 22,8 & 2,6 \\
\hline
\end{tabular}

Fonte: Elaborada pelos autores, 2020. 


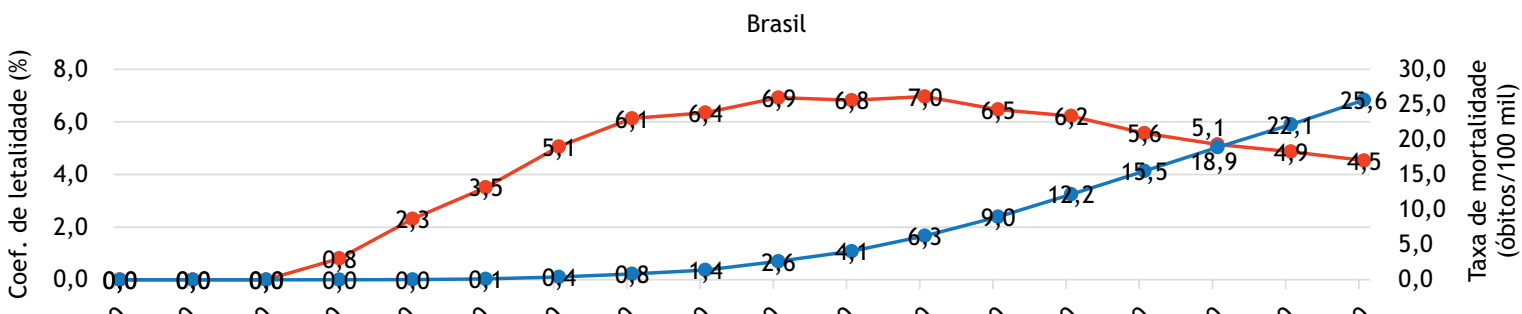

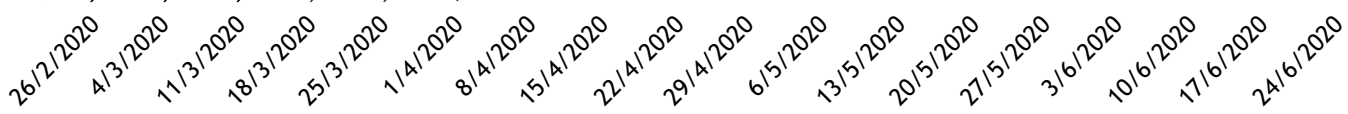

Nordeste

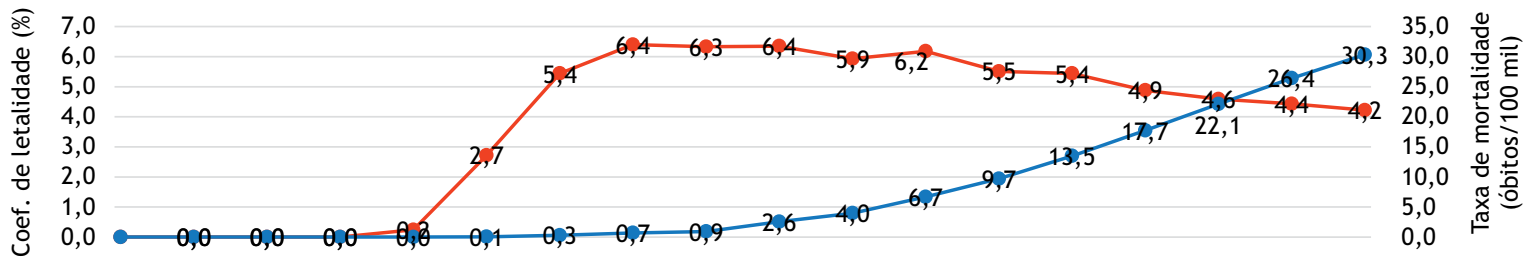

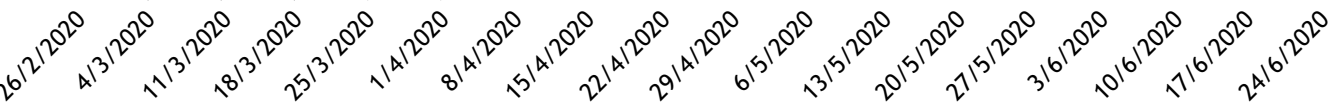

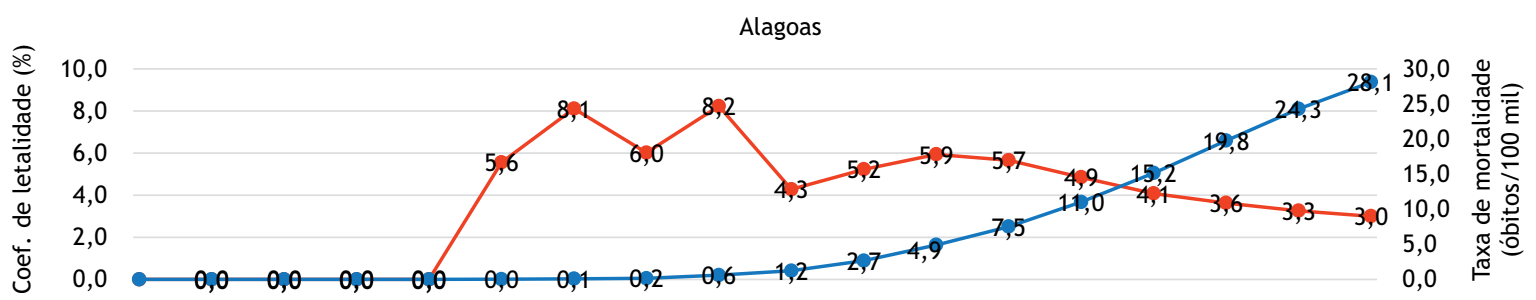

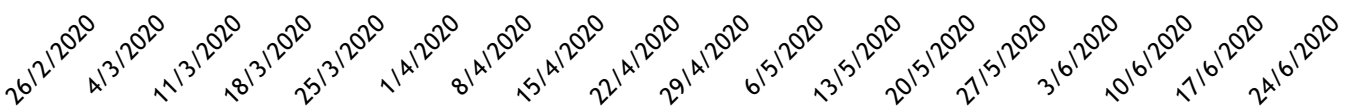

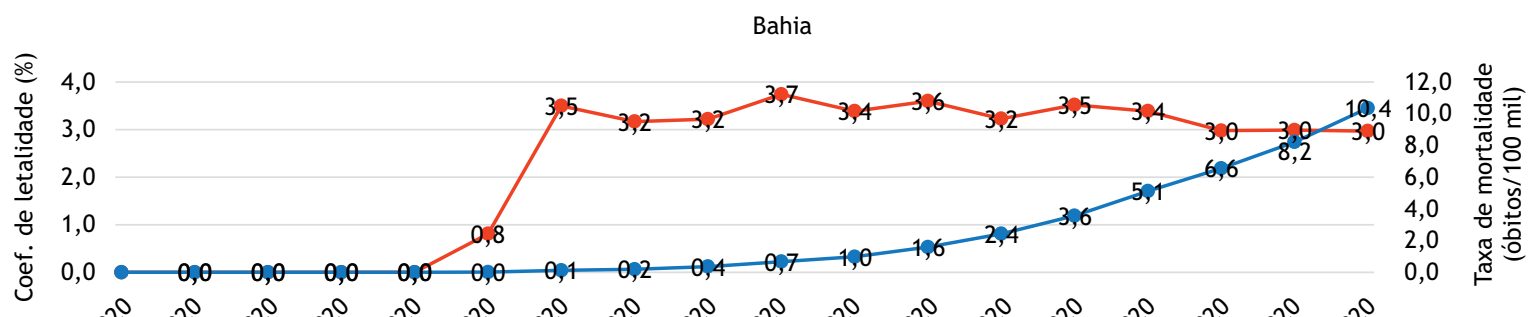

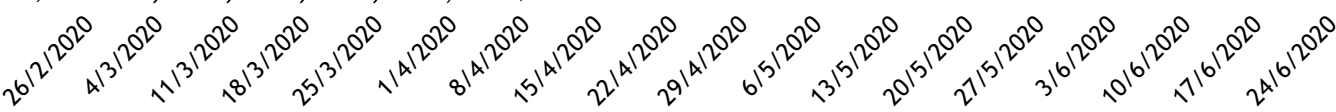

Ceará

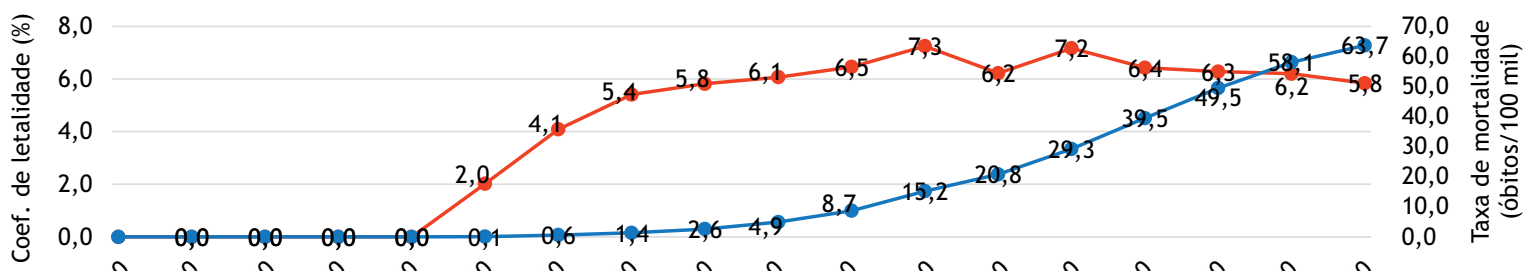

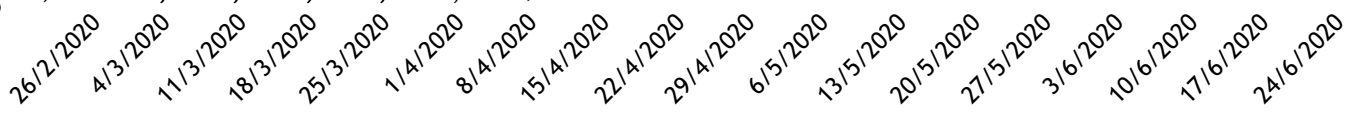

$\longrightarrow$ Letalidade $(\%) \quad \longrightarrow$ Óbitos por 100 mil hab.

Fonte: Elaborada pelos autores, 2020.

Figura. Evolução da taxa de mortalidade por 100 mil habitantes e do coeficiente de letalidade, semana a semana, no Brasil, na região Nordeste e seus estados, entre 26 de fevereiro e 24 de junho de 2020. (Continua) 


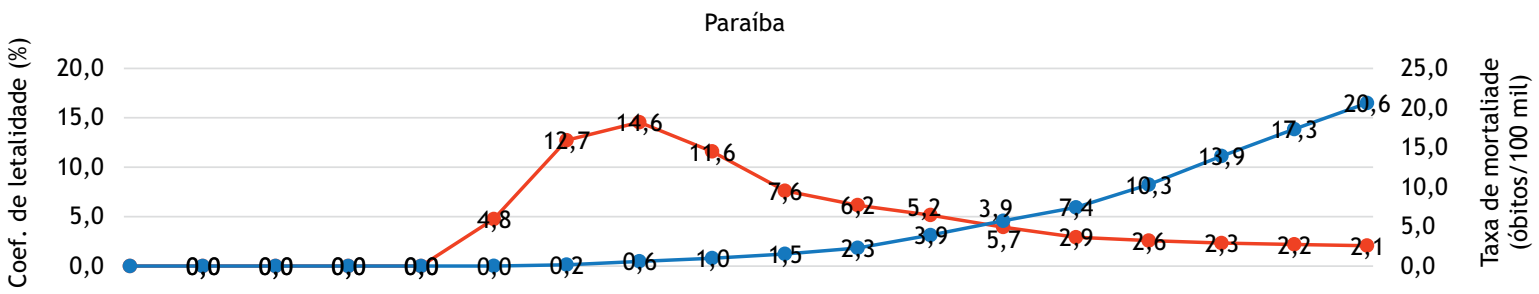

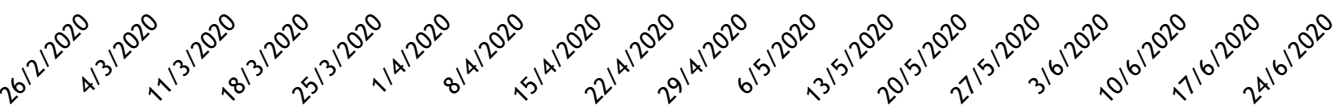

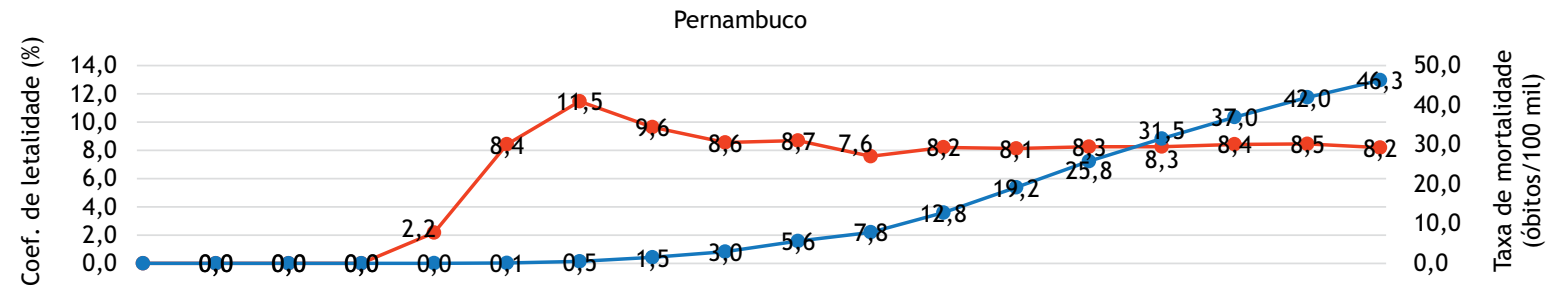

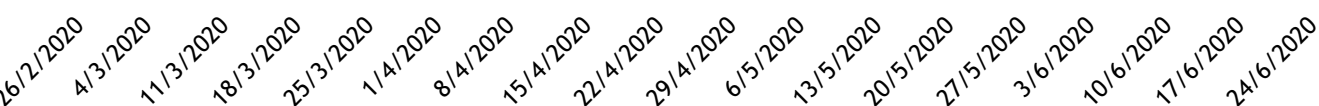

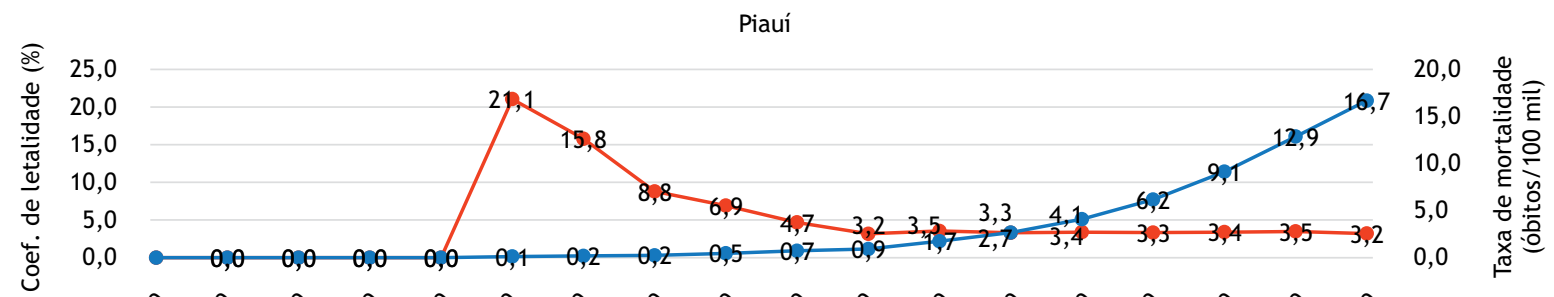

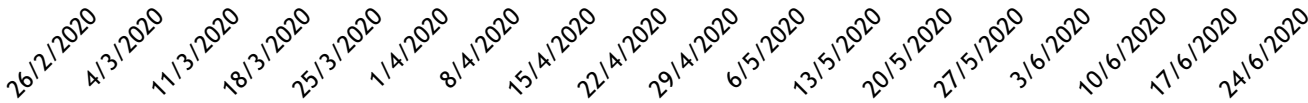

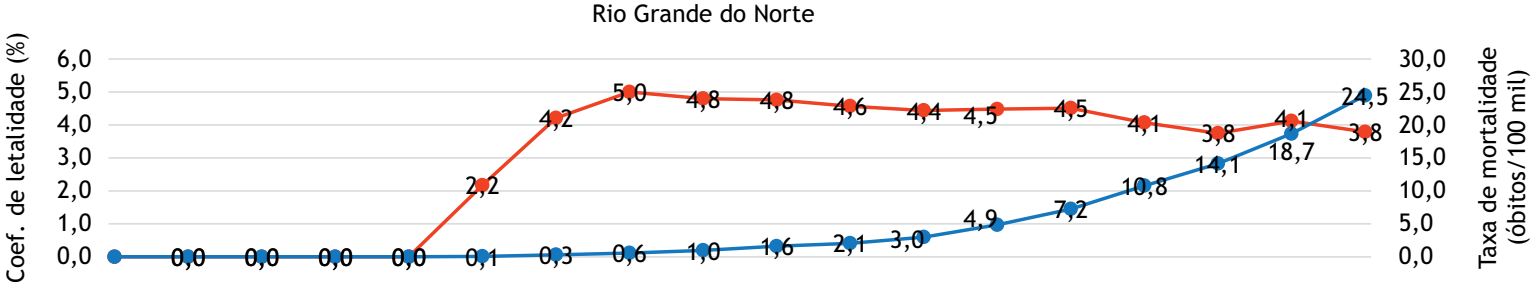

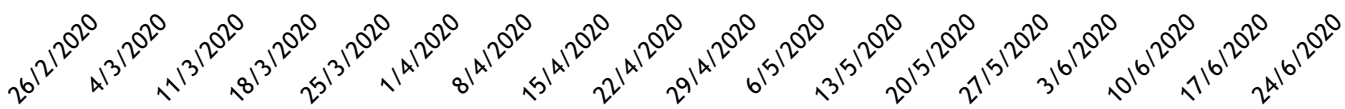

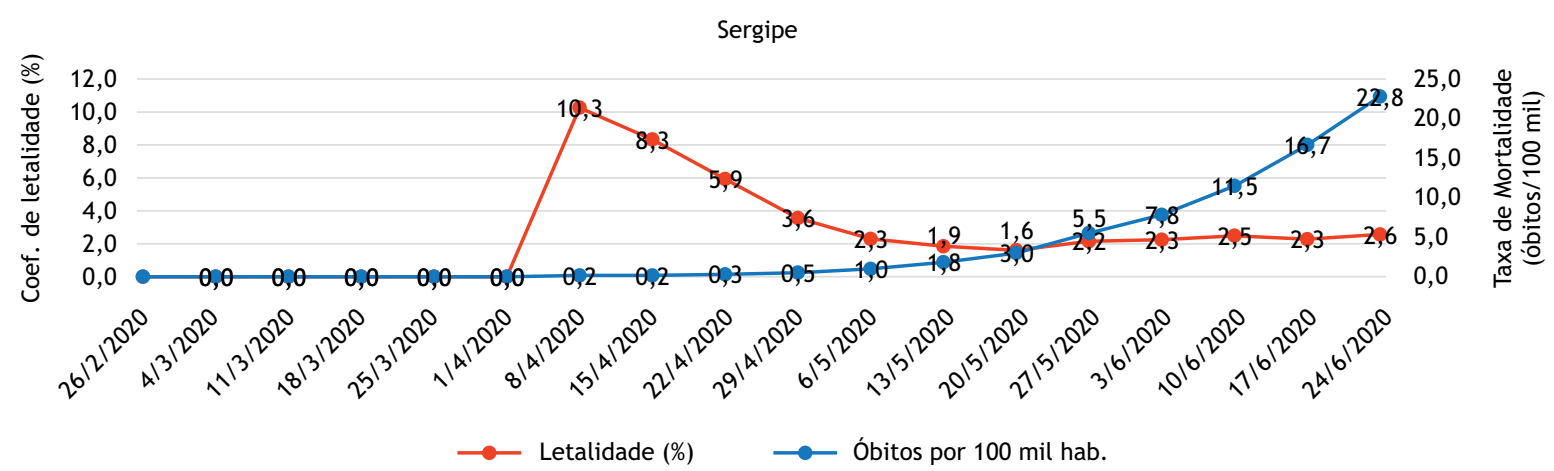

Fonte: Elaborada pelos autores, 2020.

Figura. Evolução da taxa de mortalidade por 100 mil habitantes e do coeficiente de letalidade, semana a semana, no Brasil, na região Nordeste e seus estados, entre 26 de fevereiro e 24 de junho de 2020. (Continuação) 
Tabela 2. Coeficientes de correlação entre taxas de incidência e mortalidade com os níveis isolamento social dos estados nordestinos, entre 26 de fevereiro e 24 de junho de 2020 .

\begin{tabular}{lcc}
\hline Estado & $\begin{array}{c}\text { Coeficiente de correlação entre taxa de } \\
\text { incidência e níveis de isolamento social }\end{array}$ & $\begin{array}{c}\text { Coeficiente de correlação entre taxa de } \\
\text { mortalidade e níveis de isolamento social }\end{array}$ \\
\hline Alagoas & 0,298 & 0,240 \\
Bahia & 0,176 & 0,110 \\
Ceará & 0,213 & 0,144 \\
Maranhão & 0,165 & 0,101 \\
Paraíba & 0,470 & 0,362 \\
Pernambuco & 0,232 & 0,226 \\
Piauí & 0,109 & 0,045 \\
Rio Grande do Norte & 0,207 & 0,138 \\
Sergipe & 0,346 & 0,239 \\
\hline
\end{tabular}

Fonte: Elaborada pelos autores, 2020.

embate injusto entre saúde vs. economia, cujo resultado na Itália demonstrou-se desastroso ${ }^{15}$ e ainda pior em nosso território. 0 número de casos decorrentes da pandemia ultrapassou a marca de 1,2 milhão de casos e 55 mil mortos no Brasil, pouco antes de quatro meses desde o primeiro caso confirmado 4 .

Além disso, o cenário se torna mais crítico porque as esferas governamentais não entram em consenso e, em favor da população, muitos gestores estaduais e municipais fazem uso de sua autonomia garantida por lei $\mathrm{i}^{1,15,17}$. 0 isolamento social foi pregado por todos os estados nordestinos desde o início da pandemia ${ }^{7}$, mas apenas em algumas cidades foi decretado o lockdown, como em Fortaleza e São Luís ${ }^{18}$ - localidades onde o curso da epidemia se tornou mais preocupante.

Alagoas decretou o estado de calamidade pública cerca de uma semana após o anúncio da pandemia. Desde então, o governo local somou 700 leitos clínicos e 300 leitos de terapia intensiva, até 15 de maio de 2020, e acelerou a entrega de hospitais em construção na capital e no interior ${ }^{19}$. Foi perceptível a queda da letalidade nas últimas semanas do período analisado. Contudo, esta queda pode não estar relacionada a tal investimento em sua estrutura sanitária, mas sim, devido à ampliação da testagem da população e à subnotificação de óbitos ${ }^{20}$. Importa destacar que, dentro do recorte temporal, Alagoas apresentou letalidade menor que o parâmetro de $3,4 \%$ da $\mathrm{OMS}^{10}$.

A Bahia apresenta um bom panorama em comparação com os outros estados da região, possuindo taxa de mortalidade e coeficiente de letalidade semelhantes, mesmo com alta taxa de incidência. As medidas do governo estadual incluíram a criação de 70 centros especializados para casos de COVID-19, a oferta de auxílio de $\mathrm{R} \$ 500,00$ para acometidos com quadro leve da doença, para que estes permaneçam em centros de acolhimento durante o período de transmissão, a estruturação dos serviços hospitalares e a abertura de hospitais de campanha por todo o estado $^{21}$. Houve também o decreto de isolamento social, suspensão de aulas e proibição de eventos ainda na fase inicial da pandemia no país, no mês de março ${ }^{7}$.
O Ceará é o estado nordestino com maior incidência no recorte temporal. Um estudo que correlaciona a incidência do SARS-CoV-2 na população e o Índice de Desenvolvimento Humano (IDH) na unidade federativa relata que os casos aconteceram primeiro nos bairros de maior IDH de sua capital, Fortaleza. Posteriormente, o vírus se disseminou para periferia, assolando a população mais vulnerável ${ }^{22}$. Vale ressaltar que o Ceará é o $8^{\circ}$ estado brasileiro com maior porcentagem da população vivendo na pobreza ou na pobreza extrema ${ }^{23} \mathrm{e}$ isso pode refletir negativamente no índice de isolamento social local ${ }^{7}$, entretanto o estado possui a maior média de isolamento social da região. Além dessa medida, o governo estadual aumentou o número de leitos clínicos e de terapia intensiva $(+600$ e +398 , respectivamente, até o fim de abril), ampliou a testagem e a assistência aos profissionais de saúde acometidos pela COVID-1924.

O Maranhão, segundo estado com maior número de casos no período do estudo, possui a maior porcentagem da população vivendo na pobreza e na pobreza extrema do país ${ }^{23}$ e apresenta taxa de incidência muito maior que as médias nacional e regional. Contudo, a letalidade local da doença está abaixo da média da OMS, devido à ampliação da testagem no estado ${ }^{25}$. 0 governo do Maranhão adotou medidas restritivas à aglomeração e à circulação de pessoas desde meados de $\operatorname{março}^{7}$ e, durante o desenvolvimento deste trabalho, passou a flexibilizar as medidas de isolamento ${ }^{25}$.

A Paraíba, mesmo apresentando taxa de incidência maior que a média regional e nacional, possui a menor letalidade do Nordeste. 0 governo do estado impôs medidas restritivas à aglomeração e à circulação de pessoas ainda em março, quando o estado não registrava nenhum caso da nova doença $a^{7,26}$. A ampliação da testagem e a estruturação do sistema de saúde, aliados a tais medidas de restrição, podem ajudar a melhorar os indicadores ${ }^{26,27}$.

Pernambuco foi o terceiro estado mais acometido pelo novo coronavírus, no recorte temporal. Apresentou taxa de mortalidade e coeficiente de letalidade muito elevados, mesmo sendo a 
unidade federativa do Nordeste com maior média de isolamento social. Essa medida foi decretada pelo governo estadual em meados de março, sendo decretado o encerramento temporário de todas as atividades econômicas não essenciais no início de abril7. O sistema de saúde pernambucano entrou em colapso em maio, com fila de espera para os leitos de unidade de terapia intensiva $(\mathrm{UTI})^{28}$. Nesse mesmo mês, após esse período de encerramento das atividades econômicas não essenciais, por um período de 15 dias no isolamento, o governo do estado passou a divulgar a estruturação de um "plano de convivência", passando a flexibilizar as medidas de contingenciamento no início de junho ${ }^{29}$. Nesse mesmo período, o governo do estado conseguiu zerar a espera pelos leitos de alta complexidade, após o colapso e consequente investimento em mais leitos ${ }^{30}$.

O Piauí é um dos estados com menores taxas de incidência e mortalidade e, também, o quinto menor coeficiente de letalidade do Nordeste. 0 governo iniciou a adoção de medidas não farmacológicas logo após a declaração da pandemia pela $\mathrm{OMS}^{7}$. 0 estado ainda ampliou a testagem através da criação do Programa Busca Ativa, em maio de $2020^{31}$. Logo, quanto maior o número de casos absolutos, menor será o coeficiente de letalidade ${ }^{27}$.

O Rio Grande do Norte apresentou durante o cálculo das taxas de mortalidade e letalidade, médias abaixo da regional e da nacional, mesmo fazendo parte do eixo Fortaleza/CE-Mossoró/RN que, segundo estudo de vulnerabilidade multidimensional da Superintendência do Desenvolvimento do Nordeste (Sudene), seria uma das regiões mais afetadas da pandemia no Nordeste ${ }^{32}$. 0 estado implantou medidas restritivas desde meados de març $0^{33}$, mas, dentro do recorte temporal do estudo, apresentou baixa aderência por parte da população $0^{7,13}$.

O estado de Sergipe apresentou a terceira menor taxa de letalidade da região. 0 governo local decretou as medidas de isolamento ainda em março, após a declaração da $\mathrm{OMS}^{7}$ e, desde então, tem ampliado a sua estrutura de saúde e a testagem da população ${ }^{34}$. Talvez essas medidas tenham resultado na diminuição do coeficiente de letalidade, oferecendo maior assistência à saúde e também o aumento do denominador do indicador ${ }^{27}$.

Por fim, ressalta-se que o estudo em tela apresenta limitações, por envolver a análise de dados secundários dos Sistemas de Informação em Saúde, podendo apresentar falhas na notificação dos casos. Apesar disso, acredita-se que, por se tratar de dados oficiais e de preenchimento obrigatório em todos os serviços de saúde, ainda que se considerem tais falhas, elas não anulam os resultados alcançados e o alcance dos objetivos propostos.

\section{CONCLUSÕES}

Conclui-se que o estudo das taxas de incidência e mortalidade e o coeficiente de letalidade da COVID-19 na correlação com os índices de isolamento social, por si só, não auxiliam compreender a evolução dos indicadores da pandemia da COVID-19 nos estados da região Nordeste do Brasil. E, por esse motivo, necessita-se de outras variáveis, a exemplo do nível de testagem da população e da estruturação da rede de saúde, para um melhor entendimento sobre a dinâmica da pandemia na região.

Além disso, por mais que todos os estados tenham decretado medidas de isolamento social desde o início da epidemia no país, a evolução dos indicadores entre eles variou muito. $\mathrm{E}$, mesmo onde foi decretado lockdown, como nas regiões metropolitanas de Fortaleza e São Luís, os indicares não obtiveram melhora significativa. Portanto, pode-se sugerir o caráter multifatorial dos reflexos e desdobramentos da crise da COVID19 em cada localidade.

Por fim, faz-se importante sensibilizar a população para a adoção de medidas protetivas, principalmente o isolamento social, para que se achate a curva e novos surtos não tomem as proporções do observado atualmente.

\section{REFERÊNCIAS}

1. Silva CM, Arbilla G. COVID-19: challenges for a new epoch. Rev Soc Bras Med Trop. 2020;53:1-3. https: //doi.org/10.1590/0037-8682-0270-2020

2. Li R, Pei S, Chen B, Song Y, Zhang T, Yang W et al. Substantial undocumented infection facilitates the rapid dissemination of novel coronavirus (SARS-CoV2). Science. 2020;368(6490):489-93. https://doi.org/10.1126/science.abb3221

3. World Health Organization - WHO. Who director-general's opening remarks at the media briefing on COVID-19: 11 March 2020. WHO Speeches. 11 mar 2020[acesso 17 jun 2020]. Disponível em: https://www.who.int/dg/speeches/ detail/who-director-general-s-opening-remarks-at-themedia-briefing-on-covid-19---11-march-2020

4. Ministério da Saúde (BR). Painel coronavírus. Brasília: Ministério da Saúde; 2020[acesso 17 jun 2020]. Disponível em: https://covid.saude.gov.br/
5. Carvalho ARVS, Cezarotti Filho ML, Azevedo PCP, Silveira Filho RN, Barbosa FT, Rocha TJM et al. Epidemiology, diagnosis, treatment, and future perspectives concerning SARS-COV-2: a review article. Rev Assoc Med Bras. 2020;66(3):370-4. https://doi.org/10.1590/1806-9282.66.3.370

6. Lima DLF, Dias AA, Rabelo RS, Cruz ID, Costa SC, Nigri FMN et al. COVID-19 no estado do Ceará, Brasil: comportamentos e crenças na chegada da pandemia. Cienc Saude Coletiva. 2020;25(5):1575-86. https://doi.org/10.1590/1413-81232020255.07192020

7. Aquino EML, Silveira IH, Pescarini JM, Aquino $\mathrm{R}$, Souza Filho JA, Rocha AS et al. Medidas de distanciamento social no controle da pandemia de COVID-19: potenciais impactos e desafios no Brasil. Cienc Saude Coletiva. 2020;25(supl.1):2423-46. https://doi.org/10.1590/1413-81232020256.1.10502020 
8. Garcia LP, Duarte E. Intervenções não farmacológicas para o enfrentamento à epidemia da COVID-19 no Brasil. Epidemiol Serv Saude. 2020;29(2):1-4. https://doi.org/10.5123/s1679-49742020000200009

9. Duczmal LH, Almeida ACL, Duczmal DB, Alves CRL, Magalhães FCO, Lima MS et al. Vertical social distancing policy is ineffective to contain the COVID-19 pandemic. Cad Saude Publica. 2020;36(5):1-9. https://doi.org/10.1590/0102-311×00084420

10. Marinelli NP, Albuquerque LPA, Sousa IDB, Batista FMA, Mascarenhas MDM, Rodrigues MTP. Evolução de indicadores e capacidade de atendimento no início da epidemia de COVID-19 no nordeste do Brasil, 2020. Epidemiol Serv Saude. 2020;29(3):1-9. https://doi.org/10.5123/s1679-49742020000300008

11. Faculdade de Medicina de Ribeirão Preto - FMRP. Painel COVID-19 Brasil: monitoramento e análises da situação do coronavírus no Brasil. Ribeirão Preto: Universidade de São Paulo; 2020[acesso 21 jun 2020]. Disponível em: https://ciis.fmrp.usp.br/covid19/

12. Instituto Brasileiro de Geografia e Estatística - IBGE. Portal cidades. Brasília: Instituto Brasileiro de Geografia e Estatística; 2020[acesso 21 jun 2020]. Disponível em: https://cidades.ibge.gov.br/brasil/panorama

13. Inloco. Mapa brasileiro da COVID-19. São Paulo: Inloco; 2020[acesso 21 jun 2020]. Disponível em: https://mapabrasileirodacovid.inloco.com.br/pt/

14. Shimakura S. Interpretação do coeficiente de correlação. Curitiba: Universidade Federal do Paraná; 2006[acesso 22 jun 2020]. Disponível em: http://leg.ufpr.br/ silvia/CE003/node74.html

15. Souza DO. A pandemia de COVID-19 para além das ciências da saúde: reflexões sobre sua determinação social. Cienc Saude Coletiva. 2020;25(supl.1):2469-77. https://doi.org/10.1590/1413-81232020256.1.11532020

16. Campos GWS. O pesadelo macabro da COVID-19 no Brasil: entre negacionismos e desvarios. Trab Educ Saude. 2020;18(3):1-5. https://doi.org/10.1590/1981-7746-sol00279

17. Brasil. Constituição Federal da República Federativa do Brasil. Brasília: Senado Federal; 1988.

18. Madeiro C. CE e MA apontam benefícios do lockdown e iniciam reabertura da economia. UOL Notícias Saúde. 31 maio 2020[acesso 23 jun 2020] Disponível em: https://noticias.uol.com.br/saude/ultimas-noticias/ redacao/2020/05/31/ce-e-ma-freiam-covid-19-aposlockdown-e-iniciam-reabertura-da-economia.htm

19. Governo do Estado de Alagoas. Painel interativo da COVID-19 em Alagoas. Maceió: Governo do Estado de Alagoas; 2020[acesso 24 jun 2020]. Disponível em: http://www.alagoascontraocoronavirus.al.gov.br/

20. Prado MF, Antunes BBP, Bastos LSL, Peres IT,

Silva AAB, Dantas LF et al. Análise da subnotificação de COVID-19 no Brasil. Rev Bras Ter Intensiva. 2020;32(2):224-8. https://doi.org/10.5935/0103-507X.20200030
21. Secretaria de Comunicação Social do Governo da Bahia - Secom-BA. Entenda as medidas de combate ao coronavírus na Bahia. Salvador: Governo da Bahia; 2020[acesso 24 jun 2020]. Disponível em: http://www.secom.ba.gov.br/2020/03/152898/Entenda-asmedidas-de-combate-ao-coronavirus-na-Bahia.html

22. Maciel JAC, Castro-Silva II, Farias MR. Análise inicial da correlação espacial entre a incidência de COVID-19 e o desenvolvimento humano nos municípios do estado do Ceará no Brasil. Rev Bras Epidemiol. 2020;23:1-17. https: / /doi.org/10.1590/1980-549720200057

23. Nery C. Extrema pobreza atinge 13,5 milhões de pessoas e chega ao maior nível em 7 anos. Agência IBGE Notícias. 6 nov 2019[acesso 25 jun 2020]. Disponível em: https://agenciadenoticias.ibge.gov.br/ agencia-noticias/2012-agencia-de-noticias/noticias/25882 extrema-pobreza-atinge-13-5-milhoes-de-pessoas-e-chegaao-maior-nivel-em-7-anos

24. Governo do Estado do Ceará. Confira as ações que o governo do Ceará vem promovendo no combate à pandemia. Fortaleza: Governo do Estado do Ceará; 2020[acesso 25 jun 2020]. Disponível em: https://www. ceara.gov.br/2020/06/19/confira-as-acoes-que-o-governodo-ceara-vem-promovendo-no-combate-a-pandemia/

25. Governo do Maranhão. Coronavírus Maranhão. São Luís: Governo do Maranhão; 2020[acesso 25 jun 2020]. Disponível em: https://www.corona.ma.gov.br/

26. Governo do Estado da Paraíba. Governo da Paraíba presta contas de todas as medidas tomadas até agora, durante a pandemia. Notícias. 25 maio 2020[acesso 26 jun 2020]. Disponível em: https://paraiba.pb.gov.br/noticias/governoda-paraiba-presta-contas-de-todas-as-medidas-tomadasate-agora-durante-a-pandemia

27. Pizzichini MMM, Patino CM, Ferreira JC. Measures of frequency: calculating prevalence and incidence in the era of COVID-19. J Bras Pneumol. 2020;46(3):1. https://doi.org/10.36416/1806-3756/e20200243

28. Morelli R, Ribeiro B, Resk F, Tosta F. Após Brasil bater recordes, cinco estados se aproximam do colapso na saúde. Estadão Conteúdo. 25 maio 2020[acesso 26 jun 2020]. Disponível em: https://noticias.uol.com.br/saude/ultimas-noticias/ estado/2020/05/25/apos-brasil-bater-recordes-cincoestados-se-aproximam-do-colapso-na-saude.htm

29. Secretaria de Saúde do Estado de Pernambuco SSE-PE. Governo lança plano de convivência com a COVID-19 e estabelece retorno gradual das atividades econômicas. PE Contra Coronavirus. 1 jun 2020[acesso 26 jun 2020]. Disponível em: https: / /www.pecontracoronavirus.pe.gov.br/governo-lancaplano-de-convivencia-com-a-covid-19-e-estabelece-retornogradual-das-atividades-economicas/

30. Governo do Estado de Pernambuco. Pernambuco com fila zerada de pacientes para as UTIs de COVID-19. Blog de Notícias. 7 jun 2020[acesso 26 jun 2020]. Disponível em: http://www.pe.gov.br/b/22789 
31. Governo do Estado do Piauí. Decreto № 18.984 de 20 de maio de 2020. Dispõe sobre as medidas de isolamento social a serem aplicadas nos dias 23 e 24 de maio de 2020 , estabelece o prazo para a constituição das equipes de busca ativa da COVID-19 em cada território de desenvolvimento do estado, prorroga a vigência dos decretos que especifica, e dá outras providências. Diário Oficial do Estado. 21 maio 2020.

32. Superintendência do Desenvolvimento do Nordeste Sudene. Análise multicritério da vulnerabilidade à pandemia de COVID-19 na região nordeste do Brasil. Brasília: Ministério do Desenvolvimento Regional; 2020[acesso 27 jun 2020]. Disponível em: http://sudene.gov.br/images/ arquivos/planejamento/odne/documentos/vulnerabilidadecovid19-2020-NE-benevenuto-brandao.pdf

33. Governo do Estado do Rio Grande do Norte. Medidas do governo. Portal COVID-19. 23 jun 2020[acesso 27 jun 2020]. Disponível em: https://portalcovid19.saude.rn.gov.br/ medidas/medidasdogoverno/

34. Governo do Estado de Sergipe. Sergipe contra o coronavírus: responsabilidade de todos. Todos Contra Coronavírus. 27 jun 2020[acesso 27 jun 2020]. Disponível em: https://todoscontraocorona.net.br/

Contribuição dos Autores

Moraes BQS, Machado MF - Concepção, planejamento (desenho do estudo), aquisição, análise, interpretação dos dados e redação do trabalho. Félix ICG - Concepção, planejamento (desenho do estudo), aquisição, análise e interpretação dos dados. Quirino TRL Concepção, planejamento (desenho do estudo), redação do trabalho. Todos os autores aprovaram a versão final do trabalho.

Conflito de Interesse

Os autores informam não haver qualquer potencial conflito de interesse com pares e instituições, políticos ou financeiros deste estudo.

Esta publicação está sob a licença Creative Commons Atribuição 3.0 não Adaptada.

Para ver uma cópia desta licença, visite http://creativecommons.org/licenses/by/3.0/deed.pt_BR. 